durch Silbernitrat hinzuzufügen: „überschüssig“, weil erst nach den letzten Parthien des Jods das Chlor vom Silber zu Chlorsilber gebunden wird, welches in der ammoniakalischen Flüssigkeit gelöst bleiben und erst beim Ansäuern zur Ausscheidung gelangen soll.

Verwendet man 0,127 g Jod zor titrimetrischen Bestimmung, so zeigt die Zahl der verbranchten com Natriumthiosulfats den Procentgehalt an reinem Jod durch Multiplication mit 10 direct an.

\title{
Italienische Beiträge zur Geschichte der Pharmacie und Botanik.
}

\author{
Bericht von F. A. Flückiger.
}

Die Betrachtung der Geschichte der Naturwissenschaften und der Pharmacie führt auf Schritt und Tritt in das alte Culturland Italien; es bedarf hier nicht des Nachweises, wie sich dieselben dort frühzeitig zu entwickeln begannen und namentlich auch am Ausgange des Mittelalters des grossen geistigen Aufschwunges jener Zeit, des "Rinascimento", ebenfalls theilhaftig wurden. Unter den Begründern der wissenschaftlichen Botanik gehören einige der bedeutenderen Italien an und deutsche "Väter der Botanik“ pilgerten auch wohl über die Alpen, um ihr Wissen zu vertiefen. Derjenige unter den letzteren, welcher der Pharmakognosie insbesondere am besten gedient hat, Valerius Cordus, hat $\mathbf{1 5 4 4}$ in Rom seine heute allerdings nicht mehr kenntliche Ruhestätte gefunden.

Es mag hier ferner unerörtert bleiben, ob die politische Zerrissenheit Italiens jenen Aufschwung gefördert oder gehemmt habe; was aber die berufsmässige Stellung der Pharmacie betrifft, so liegt es nahe, einen Vergleich anzustellen mit den um jene Zeit nicht minder zerfahrenen deutschen Landen. Wie sehr hier Magistrate und weltliche wie geistliche Fürsten der zahllosen kleinen Staatengebilde im XV. bis XVIII. Jahrhundert der Apotheke ihre gesetzgeberische Fürsorge angedeihen liessen, wird bezeugt durch die unabsehbare Reihe von bezüglichen Verordnungen, welche wohl der Mehrzahl nach schon von vornherein mit Hülfe der Presse zur Veröffentlichung gebracht wurden. Aehnliche politische Zustände mussten auch in Italien eine entsprechende reiche Entwickelung der pharmaceutisch-medicinischen Gesetzgebung hervorrufen. Von diesen Erwägungen ausgehend und mit einigen bezüglichen Werken bereits bekannt, wurde ich doch überrascht durch den vielfach interessanten 
Inhalt der hiernach genannten Schriften, welche ich der freundlichen Aufmerksamkeit ihrer Verfasser verdanke.

Wahrscheinlich finden diese Abhandlungen nicht eine sehr grosse Verbreitung im Auslande; ich hoffe daher nichts überflüssiges zu unternehmen, wenn ich die wesentlichen Ergebnisse fleissiger und geschickter Arbeit italienischer Collegen in diesen Blättern beleuchte.

Obwohl man bei Betrachtung der älteren Pharmacie in Italien gewöhnlich von dem Eindruck begleitet wird, dass ähnliche Ursachen dort ähnliche Wirkungen zur Folge haben mussten wie anderswo, scheinen doch dort weniger eingreifende Bestimmungen über den Verkaufswerth der Drogen und Arzneien erlassen worden zu sein als z. B. in Deutschland, wo zahlreiche Städte und Fürsten gelegentlich oder regelmässig ihre besonderen Arzneitaxen aufstellten, aus welchen uns ein lebensvolles Bild pharmaceutischer Vergangenheit entgegentritt. Möglich, dass eingehendere Forschungen auch in Italien noch dergleichen zu Tage fördern, doch vermisst man sie einstweilen in den folgenden Schriften und in den darin vorkommenden literarischen Nachweisen, welche uns gleichwohl zur Genüge zeigen, wie sehr die italienische "Storia patria " auch die Pharmacie aufmerksam berücksichtigt.

\section{I.}

Documenti storici spettanti alla medicina, chirurgia, farmaceutica conservati nell' archivio di stato in Modena. I. Serie, Elenco dei documenti esposti a Modena, durante il X. Congresso dell' associazione medica Italiana e facsimili di 24 antografi. - II. Serie, Notizie e pubblicazione di molti altri, appartenenti ai sec. XIV. e $\mathrm{XV}$. offerte oggi all' XI. Congresso della stessa associazione in Perugia. Modena, $1885.114 \mathrm{pp} .8^{\circ}$.

Der Director des Staatsarchivs in Modena, Cav. Cesare Foucard, hat, wie die Überschrift besagt, im September 1882, bei der Zusammenkunft des italienischen Aerzte-Vereines in Modena eine Ausstellung von Documenten veranstaltet, welche sich auf die Geschichte der Medicin, Chirurgie und Pharmacie, hauptsächlich im XV. Jahrhundert, beziehen. Nicht am wenigsten Interesse dürfen die Nachbildungen der Unterschriften von 24 berühmten italienischen Aerzten aus der Zeit von 1461 bis 1785 in Anspruch nehmen, darunter z. B. Antonio Musa Brassavolo, Marcello Malpighi, Lazaro Spallanzani. 
Als sich der genannte Verein 1885 in Perugia versammelte, legte Foucard demselben ein umfangreiches Verzeichnis ähnlicher Documente des XIV. und XV. Jahrhunderts aus dem Archive von Modena vor.

Die vorliegende Schrift nennt in fortlaufenden Nummern den Inhalt der betreffenden Schriften; von pharmaceutischem Interesse sind darunter die nachstehenden. No. 61 und 62 Recept zu einem „Balsamo artifficiato", dessen Ingredientien aber hier nicht angegeben sind. No. 62 Recept des XIV. Jahrhunderts zu einer Species gegen Hüftschmerzen (male del fiancho che procede da renello); unter den 20 Bestandtheilen sind höchstens hervorzuheben Cubeben, Muscatnuss, indische Nuss (Cocos). Man sieht also, dass damals, wie es scheint in Bologna, die Cubeben auch als Heilmittel, nicht wie sonst als Gewürz dienten. ${ }^{1}$

No. 63, vom Jahre 1440, gedenkt einer Rechnung über Arzneien, welche in Venedig durch den Arzt Benzi für den Markgrafen Nicolò III. d'Este gekauft wurden; leider werden sie hier nicht genannt. Ebensowenig die Vorräte, welche 1578 bei der Visitation einer Apotheke (Spezieria) in Modena vorgefunden wurden.

No. 219 gibt den vollständigen Text eines Vertrages, wodurch sich der Apotheker, Magister Jacobus speciarins filius, Arivabene de Arivabene am 23. Februar 1424 in Ferrara vor Notar und Zeugen verpflichtete, während 2 Jahre die im Vertrage genannten Arzneien und Drogen zu den dort beigesetzten Preisen dem Markgrafen Nicolaus von Este zu liefern. Aus dem umfangreichen Verzeichnisse seien hier nur die folgenden genannt. Acacia (d. h. ohne Zweifel Succus Acaciae ${ }^{2}$ ) das Pfund zu 2 Soldi, Aloë hepatica 17 Soldi, Quecksilber 12, Sublimat 18, Ammoniacum 14, gesponnene Baumwolle 7 , Butter 2, Zimmt 14, Zucker 6, Cassia in canna (Cassia fistula) 5, Cassia tracta ${ }^{3} 10$, Cubeben das Pfund 1 Pfund (libre) und 16 Soldi, Camamilla das Pfund 2 Soldi, Confectio a vermibus 6 Soldi 6 Denarii, Campher das Pfund 4 Libre, Traganth das Pfund 10 Soldi, Indigo (Endeghum) das Pfund 1 Libre 16 Soldi, Muscatnuss das Pfd. 18 Soldi, Orpiment 12, Pfeffer 10, Requilicia (Süssholz) 1 Soldo 6 Den., Sementina (Flores Cinae) 16 Soldi, Senf 1, Rotes Sandel-

1) vergl. Flückiger, Pharmakognosie 1883. 875.

2) siehe Flückiger, Die Frankfurter Liste, Archiv der Pharm. 201 (1872) 461 oder S. 31 des Separat-Abdruckes.

3) mir unbekannt; vielleicht die Pulpa der Cassia fistula? 
F. A. Flückiger, Italien. Beiträge z. Gesch. d. Pharmacie u. Botanik.

holz das Pfd. 1 Lib. 4 Soldi, Safran die Unze 5 Soldi 6 Den., Ingwer das Pfd. 18 Soldi, Zucker 6 Soldi.

Dieser Vertrag, welcher einigermassen Einblick in die Wertverhältnisse einer Anzahl von Drogen gewährt, wurde wiederholt, und zwar, wie es scheint, mit geringen Änderungen erneuert. 1433 kamen unter anderm hinzu Fusti garofalorum, Nelkenstiele ${ }^{1}$, das Pfd. zu 8 Soldi, Gardamomos alexandrinos zu 1 Lib. 16 Soldi, Melegetas $^{2}$ zu demselben Preise, Seife zu 8 Soldi, wogegen unter anderem Acacia und Zucker fehlen. - Auf Bäder und Mineralwasser bezïgliche Documente sind mehr nur von localem Interesse.

\section{II.}

A. Corradi. Gli antichi statuti degli Speziali. Brano di storia della Farmacia. Milano, 1886. 77 p. gr. $8^{\circ}$. - (Alte Apothekerordnungen, Beitrag zur Geschichte der Pharmacie.) Abdruck aus Annali universali di Medicina. Vol. 277.

Aus Luigi Manzoni, Bibliografia statuaria e storica italiana, Bologna 1879, ist ersichtlich, dass in der Bibliothek der Grafen Malvezzi in Bologna eine Apothekerordnung dieser Stadt vom Jahre 1377 aufbewahrt wird und dass solche "Statuti“ oder "Capitoli“" der Stadt Siena von 1355 und der Stadt Ferrara, welche letztere sich von 1381 bis 1793 erstrecken, in den betreffenden Bibliotheken zu finden sind. Cesare Foucard hat ferner 1859 schon veröffentlicht den Statuto dei Medici e degli Speziali von Venedig aus dem Jahre 1258 und Isnardi druckte 1861 in seiner Geschichte der Stadt Genua die Capitula artis Aromatariorum sive Spectiariorum (Apotheker) comunis Januae von 1488 ab.

Schon diese wenigen Beispiele berechtigen zu der Voraussetzung, dass die Magistrate oder Fürsten der blühenden italienischen Städte des Mittelalters sich der Regelung der pharmaceutischen und medicinischen Angelegenheiten eifrig angenommen haben und bei der Sorgfalt, welche dort derartigen Documenten gewidmet zu werden pflegt, lässt sich erwarten, dass die reichen Archive und Bibliotheken Italiens noch viele, vermutlich die meisten der vom Mittelalter bis zur Neuzeit erlassenen Apotheker -Gesetze unter ihren bewunderungswürdigen Schätzen bergen.

1) Flückiger, Pharmakognosie 1883. 764.

2) ebenda 855.872 . 
Professor Corradi in Pavia, der Verfasser der vorliegenden Schrift, zählt hier derartige gedruckte und ungedruckte Verordnungen auf, um zu einer vollständigen Ausmittelung derselben den Anstoss zu geben. In diesem vorläufigen Verzeichnisse sind schon vertreten Bologna, Brescia, Cremona, Ferrara, Florenz, Genua, Lucea, Mailand, Mantua, Neapel, Novara, Padua, Palermo, Parma, Pistoja, Perugia, Pisa, Rom, Siena, Venedig, Verona, Vicenza, allerdings schon eine stattliche Zahl der edelsten Gemeinwesen der städtereichen Italia.

Der Statuto dell' Arte degli Speziali di Pisa vom Jahre 1497 führt uns die dortigen Apotheker als eine geschlossene Corporation, "L'Arte", vor, an deren Spitze 3 Consuln und 2 Räte, Taratori, standen; aus anderen Städten sind noch weiter ausgebildete derartige Einrichtungen bekannt. Die Taratori führten eine sehr weitgehende Aufsicht über die Beschaffenheit und Preise der Drogen und Präparate der Apotheken. Der Apotheker-Verein, "L'Università dell' Arte degli Speziali" hatte das Recht, jeden Standesgenossen zum Beitritte anzuhalten; wer nicht die Eintragung in die Matrikel bezahlte, verfiel einer hohen Geldbusse. In manchen Städten gehörten noch zu der "Apothekerkunst" Drogisten aller Art, Feinbäcker, Zuckerbäcker, Destillateure und andere Vertreter der in Italien von jeher so reich entwickelten Fabrication feinerer Genussmittel; derartige Bundesgenossen der Pharmacie werden oft unterschieden als Sottoposti oder Obbedienti. Bisweilen aber werden auch die Apotheker ihrerseits den Gilden der Kaufleute oder der „Università dei Medici" zugetheilt.

Im XV. Jahrhundert waren auch in Rom die Apotheker zunftmässig vereinigt und besassen in S. Lorenzo in Miranda eine kleine, ihnen 1430 vom Papste Martin .V. überlassene Kirche, wo sie ein Armenhaus (Spedale pei poveri della loro arte) errichteten. ${ }^{1}$

Für die italienische Localgeschichte wie für die Geschichte der Pharmacie im allgemeinen wird ein vergleichendes Studium aller dieser Verhältnisse sehr wertvoll sein. Unter dem Schutze der städtischen Freiheit, wie im Schatten der blutigen Händel zwischen den zahlreichen fürstlichen und republicanischen Gemeinwesen scheinen es die Apotheker bisweilen verstanden zu haben, sich ein recht

1) Pasquale Adinolfi. Roma noll' età di mezzo. I (1881) Cap. XXI, $\S 11$, p. $416-418$. 
bedeutendes Ansehen zu sichern. Dazu trug wohl z. B. in Pisa gerade zu Ende des XV. Jahrhunderts das nnglückselige Verhältnis dieser Stadt zu dem "magnifico popolo di Firenze" mächtig bei. Mit erstaunlicher Vollständigkeit war die Pharmacie in Pisa durch die umständlichsten Verordnungen geregelt. An den gebotenen Festtagen blieb auf jeder Seite des Flusses nur eine Apotheke geöffnet; erst nach der 23. Stunde durften die übrigen ohne Aufsehen zu erregen das Guckfensterchen, lo sportello, öffnen. Jeder Berufsgenosse musste in genau vorgeschriebener Weise am 27. September das Fest der Schutzpatrone der Pharmacie, Cosma und Damian, so wie am 15. August, in dem wundervollen Dom Pisas, den Himmelfahrtstag, Assunzione della Madonna, mitmachen.

Die Consuln dell' Arte hatten das Recht und die Pflicht, jede Apotheke vierteljährlich einer Besichtigung, cierca, zu unterwerfen; auch die Vorräte der oben 'genannten zugehörigen Gewerbsleute wurden geprüft. Mit ganz besonderer Sorgfalt wurden in Pisa, wie ja auch anderswo, der Theriak und andere Latwergen überwacht, deren richtige Mischung sich allerdings mit den damaligen Hülfsmitteln nachträglich nicht feststellen liess. Unter den verpönten Zusätzen werden genannt Esula (Euphorbia-Arten), Catapuzia (Euphorbia Lathyris) und Helleborus. Der religiöse Zeitgeist machte die Herstellung von Wachslichtern und Fackeln zu einem für manchen Apotheker erwünschten Nebengewerbe; auch dieses war genau geregelt und jedes Stück musste den Stempel der betreffenden Apotheke tragen. Daher war denn auch zu Dochten geeignete Baumwolle ein Verkaufsartikel der Apotheken.

Für eine Reihe von andern war die dem Käufer zu gewährende Tara durch die oben genannten Taratori genau vorgeschrieben. Unter diesen mögen hervorgehoben werden Trementina in otri (in Schläuchen) und Trementina veneziana; erstere ist wohl als der ursprüngliche Terpenthin aus Chios und Cypern zu deuten, welcher also um diese Zeit (1497) in Pisa noch zu haben war. ${ }^{1}$

Nur zwischen dem 15. October und 15. April durften gewisse Backwerke angefertigt werden, damit dergleichen in der heissen

1) Flückiger and Hanbury, Pharmacographia, 2d edition 1879 , p. 609. Eine frühere ausdrückliche Erwähnung der Terebinthina veneta ist mir nicht bekannt. 
Jahreszeit gar nicht oder doch weniger genossen würden, indem wohl Angesichts der sumpfigen Umgebung Pisas zur Sommerszeit die Gefahr von Erkrankungen durch jene Leckereien gesteigert werden konnte.

Corradi ermöglicht eine Vergleichung dieses Pisanischen Statuto von 1497 mit den entsprechenden Verordnungen von Bologna (1377) und Genua (1488), indem er die Überschriften ihrer einzelnen Capitoli anführt. Capitel V des genuesischen Statuts bestimmt, "quod nullus speciarius faciat apothecam vel societatem cum aliquo medico in dicta arte" und Capitel XXV: „quod nullus speciarius doceat suam artem aliquem sclavum ".

Das schon (wahrscheinlich 1231 oder 1232) von Kaiser Friedrich II. erlassene Verbot ${ }^{2}$ einer Verständigung des Arztes mit dem Apotheker (confectionarius) findet sich auch im Jahre 1258 in einem Capitolare von Venedig, noch nachdrücklicher aber wurde in Perugia 1378 untersagt, dass der Apotheker (Speziale) dem Arzte gebe "aliquam provisionem ut moretur et stet in camera ipsius spetiarii“.

Die zunftmässige Selbständigkeit, zu welcher sich bisweilen die Apotheker, die „Università degli Speziali“ einer Stadt vereinigten, hatte notwendigerweise eine gewisse Engherzigkeit, Überhebung und Ausschliesslichkeit im Gefolge. Solchen Übelständen suchten manche Bestimmungen die Spitze abzubrechen, indem z. B. den Mitgliedern der Università nach verschiedenen Richtungen gegenseitige Rücksichten auferlegt wurden. Die Apothekergenossenschaft in Ferrara erhielt 1409 vom Markgrafen Ni colò III. d'Este ihre Statuti, deren Capitolo 3 die Apotheker anwies, nötigenfalls für die Beerdigung der Genossen zu sorgen; Capitolo 5 betraf die Unterstützung verarmter Collegen. Diese Compagnia degli Speziali in Ferrara blühte bis in das vorige Jahrhundert; 1738 widmete sie den Schutzpatronen S. S.

1) Angesichts des bis in das XV. Jahrhundert von den italienischen Kaufleuten gelegentlich betriebenen Sclavenhandels, welcher bisweilen Sclaven und Sclavinnen nach Genua, Venedig $u$. s. w. führte, erscheint das obige Verbot wohl gerechtfertigt. Bekanntlich erachtete es selbst Lorenzo il Magnifico, der grosse Beschützer der Kunst und Wissenschaft, nicht unter seiner Würde, sich Sclavinnen zuführen zu lassen. - Vergl. auch Heyd, Levantehandel im Mittelalter II (1879) 543: Menschen (Sklaven).

2) Winkelmann, Acta imperii inedita 1880, 791; im Auszuge auch Archiv der Pharmacie Bd. 83 (1843) 195: Constitutiones Neapolitanae sive Siculae, Tit. XXXIV. 
Cosma und Damiano eine kleine aber elegante, heute noch bestehende Kirche, während die „Universită“ der ferraresischen Apotheker längst eingegangen ist.

Die erste im Druck erschienene Apothekerordnung ist wohl das "Breve milanese", welches 1496 bei Leonardo Pachel in Mailand die Presse verliess. - Im XVI. Jahrhundert behauptete sich dieses Gesetzbuch ziemlich unverändert bis 1573 in 3 Auflagen unter dem Titel "Constitutiones Statuta et Ordinamenta Paratici ${ }^{1}$ et Universitatis Aromatariorum sive Speciariorum Civitatis et suburbiorum Mediolani". 1573 lautete der Titel Privilegium Aromatariorum etc.; unter den 54 Capiteln dieser Ordnung schreibt das 17. vor "quod Aromatarii debeant tenere libros bene ordinatos " und das folgende handelt "de denuntiatione alterius Aromatarii". Nummer 51 verbietet Piperino and $Z$ afloro als verwerfliche Surrogate des Pfeffers und des Safrans.

Im 5. Bande des Periodico della Società Storica Comense findet sich die dem mailändischen Breve offenbar nachgebildete Apothekerordnung der Stadt Como vom Jahre 1514: "Statuti Paratici et Universitatis Aromatariorum Comi", beginnend mit der Anrufung der Madonna und Sant' Abondio's, des Schutzheiligen der Stadt. Die 69 Paragraphen dieser Ordnung, welche auf einer ältern zu beruhen scheinen, werden ohne Nummern aufgeführt. Der Vorstand der Apothekergenossenschaft Comos wurde gebildet aus einem durch den Titel Abbate geehrten Präsidenten, 2 Consuln, einem Canovajo und 2 Sindachi. Dem mit einem geringen Gehalte bedachten Abbate standen beträchtliche Befugnisse zu, obwohl er nur auf ein Jahr gewählt und erst nach Verfluss von 2 Jahren wieder wählbar wurde. Das Original dieser Statuti von Como ist ein prachtvolles, mit Miniaturen versehenes Manuscript, welches im Staatsarchiv zu Mailand liegt. Dass dieselben der Hauptsache nach mit den entsprechenden Ordnungen anderer italienischer Städte jener Zeit übereinstimmen, versteht sich. Es würde aber sicherlich von Interesse sein, bezügliche Vergleichungen in grösserem Umfange anzustellen und auch andere Länder herbeizuziehen.

III.

Illustrazione del ducale Er bario Esten se conservato nel R. Archivio di Stato in Modena. Da J. Camus, Professore nella R. Scuola

1) so viel als Zunft, Gilde, Verein von Ansehen (Paragium bei Du Cange). 
militare ed O. Penzig, Direttore della R. Stazione agraria di Modena. Modena 1885,46 pp. $8^{\circ}$. (Estr. dagli Atti della Società dei Naturalisti di Modena, Serie III, Vol. IV.)

Als Cesare d'Este im Jahre 1598 sein Herzogtum Ferrara dem Papste Clemens VIII. überlassen musste und nach Modena übersiedelte, nahm er unter andern Sammlungen des kunstliebenden Hauses Este auch ein Herbarium mit, welches jetzt im Staatsarchive in Modena aufbewahrt wird. Nach sorgfältiger Untersuchung konnten die Verfasser der vorliegenden Schrift feststellen, dass die Pflanzen desselben zwischen 1585 und 1598 in der Umgebung von Ferrara gesammelt worden sein müssen; vereinzelte Anzeichen sprechen dafür, dass vielleicht schon vor 1560 eine solche Sammlung in Ferrara angelegt worden wäre. Der Botaniker, welchem die Nachwelt für das uns glücklicherweise erhaltene „Erbario Estense" verpflichtet ist, scheint wohl kein Gelehrter, sondern eher ein Gärtner gewesen zu sein. Italien besitzt allerdings heute noch von 2 seiner ausgezeichnetsten Botaniker, Andrea Cesalpino in Pisa und Ulisse Aldrovandi in Bologna bedeutende Herbarien aus dem XVI. Jahrhundert, neben welchen aber dieses Estensische ebenfalls, durch die Untersuchungen von Camus und Penzig, seine Stelle beanspruchen darf.

Diese schliessen sich der Ansicht Meyer's ${ }^{1}$ an, dass der Engländer John Falconer, der kurz vor dem Jahre 1547 in Ferrara weilte, der erste war, welcher Pflanzen trocknete, also wohl zuerst den Anstoss zur Anlegung von Herbarien gab. Denn dasjenige von Aldrovandi geht bis 1554, dasjenige des Arztes Greault in Lyon bis 1558 zurück und das Herbarium Cesalpin i's gehört in das Jahr 1563, wie hier nachgewiesen wird; das Aldrovandi'sche, aus 16 Bänden bestehend, harrt noch der Bearbeitung, während Caruel schon 1858 dem Herbarium Cesalpini's eine gründliche Untersuchung gewidmet hat. Nimmt man noch dazu die Herbarien von Rauwolf in Leiden und das in Basel aufbewahrte Bauhin'sche, so wird durch diese Herbarien wohl so ziemlich der Gesamtbestand an getrockneten Pflanzen aus dem XVI. Jahrhundert bezeichnet sein. Der Beitrag aus dem ehemaligen herzoglich Este'schen Besitze darf daher wohl mit Recht als willkommen begrüsst werden.

1) Geschichte der Botanik, IV (1857) 270. 
Dass sich übrigens jene Familie auch noch in anderer Weise im Geiste der Zeit des Wiedererwachens der Wissenschaft einiges Verdienst um die Botanik erworben hat, zeigen die Verfasser der vorliegenden "Illustrazione", indem sie aus den Schriften der herzoglichen Kanzlei (im Archive zu Modena) ein freundschaftliches Schreiben abdrucken, welches Alfonso II. d'Este am 31. Juli 1574 aus Ferrara an den türkischen Minister Mehemet Pascha erliess, um diesen zu bitten, dem Herzoge Holz, Früchte und Oel des Balsambaumes ${ }^{1}$ und andere zur Bereitung des Theriaks ${ }^{2}$ erforderliche Stoffe zu verschaffen. Ferner bat der Herzog um Samen von solchen Arzneipflanzen, welche etwa in seinen Gärten zur Blüte gebracht werden könnten und erbot sich zu Gegendiensten. Schon der Vater und der Ahnherr Alfonso's, Ercole II. und Alfonso I. hatten in den ersten Jahrzehnten des XVI. Jahrhunderts schöne Gärten in Ferrara und eine Orangerie auf einer Insel im Po angelegt. Als sich A mato Lusitano ${ }^{3} 1540$ bis 1547 in Ferrara aufhielt, rühmte er bewundernd die Pracht der Gärten des dortigen reichen Einwohners Acciajuoli, aus welchen Amatus Material zum Unterricht in der Pharmakognosie, "per l'insegnamento dei semplici", bezog. 1542 sandte König Ferdinand aus Prag seinen Gärtner Claudius Reynart nach Italien, um die berühmten Gärten, besonders die ferraresischen zu sehen, damit er in Stand gesetzt werde, den um das Jahr 1537 in Prag angelegten königlichen Garten zu verschönern.

Im Archive zu Modena liegt ein wahrscheinlich um die Mitte des XVI. Jahrhunderts, vielleicht von einem deutschen Gärtner, verfasster Catalog des herzoglichen Pavillon-Gartens, welchen die Verfasser in der vorliegenden Schrift abdrucken liessen. Neben Zierpflanzen, wie z. B. Hyacinthen, Jonquillen (Narcissus Jonquilla L., siehe Archiv der Pharmacie Bd. 222, 1884, p. 481), Lilac, Tulipa, weist dieser Catalog auch Digitalis flore purpureo, so wie Solatrum majus (Atropa Belladonna?) auf. - Der Name Digitalis rührt meines Wissens von Leonhard Fuchs (1542) her*,

1) Balsamea meccanensis Gleditsch (Balsamodendron gileadense Kunth); siehe Flückiger, Pharmakognosie 130.

2) dieses hochberühmte Electuarium wurde bekanntlich mit besonderer Virtuosität in Venedig bereitet, daher wohl das Interesse des Herzogs an diesem Präparate.

3) Flückiger 1. c. 986.

4) ebenda 668 . 
spricht also hier dafür, dass dieser Catalog nach 1542 verfasst sein muss.

Nach diesem letzteren folgt das Verzeichnis des herzoglichen Herbariums mit den Namen, welche die Pflanzen in demselben führen; diese sind von den Verfassern in die Sprache der heutigen Systematik übersetzt und mit lehrreichen kritischen Bemerkungen versehen worden. Die Namen des Herbariums sind nicht selten schon aus zwei Wörtern gebildet, wie es später durch Linné zur Regel gemacht wurde.

Aus diesem Herbarium sind bemerkenswert: Lauro gregio, Prunus Lanrocerasus, zwar nur noch als Abdruck auf dem Papier erhalten, doch mit aller Sicherheit kenntlich; auch in dem oben erwähnten Caesalpin'schen Herbarium findet sich Laurocerasus unter dem Namen Lauro regio. Der Kirschlorbeer ist zwischen 1546 und 1549 von Pierre Belon bei Trapezunt gefunden und durch ihn in Europa bekannt geworden. ${ }^{1} 1550$ war Belon wieder in Paris, wo 1553 sein Reisebericht, Observations etc., erschien. Unmöglich kann also wohl Prunus Laurocerasus vor dieser Zeit in das Estensische Herbar gelangt sein; durch Belon selbst wissen wir, dass der Kirschlorbeer z. B. $1558 \mathrm{im}$ Garten von Pisa vorhanden war. ${ }^{2}$

Für die geringe Zuverlässigkeit mancher Benennungen des Herbars spricht die Bezeichnung Phu oder Valeriana minor, welche dort dem Thalictrum angustifolium L. beigelegt ist. Von einer Herba, welche der berühmte genuesische Admiral Andrea Doria, nach Aussage des Herbariums, in Italien eingeführt habe, ist leider nur noch ein Blatt übrig, welches sich nicht mehr bestimmen lässt. ${ }^{3}$

Die historisch bemerkenswerteste Pflanze des Erbario Estense ist wohl Tabacho oder Herba Regina, Nicotiana Tabacum; wahrscheinlich gibt es keine älteren Blätter und Blüten dieser Art als die hier liegenden. ${ }^{4}$ Unter "Sena vera delle spiciarie che vien di Levante", Sennesblätter der Apotheken, finden sich Blätter und Früchte, welche von den Verfassern der Cassia obovata Colladon zugeschrieben werden. Die Hülse dieser Art ist so auffallend, dass sie nicht zu verkennen ist; ausserdem war ja Cassia obovata damals in Tos-

1) Flückiger 1. c. 989 .

2) ebenda 727 .

3) ob wohl versucht worden ist, das Microscop zu Hülfe zu nehmen?

4) Flückiger 1. c. 679. 
cana in grösserem Masstabe angebaut. ${ }^{1}$ Wenn dagegen auch ein Fruchtstand des Nelkenbaumes, Eugenia caryophyllata, im Herbar liegt, so darf wohl nicht etwa auf Cultur desselben geschlossen werden, denn die "Nelkenstiele", Fusti oder Bastaroni di garofali, bildeten ja während des ganzen Mittelalters bis zur Gegenwart einen bedeutenden Artikel des Levantehandels der italienischen Seestädte. ${ }^{2}$

Masturtio oder Nasturtio d'India, Blätter und Blüten des aus Peru stammenden Tropaeolum minus L., dürften ebenfalls die ältesten Exemplare dieser Zierpflanze sein, welche 1596 in Pisa eingeführt wurde, aber allerdings schon früher Monardes und Clusius bekannt war. Auch der Blütenzweig der "Pomi di Etiopia oder Pomi d'oro", Solanum Lycopersicum, gleichfalls aus Peru, verdient Beachtung; Mattioli gedenkt ihrer ebenfalls.

Ein Register der italienischen im Erbario Estense vorkommenden Namen und der von den Verfassern ausgemittelten systematischen Bestimmungen gibt ihrer "Illustrazione" vollen Wert als Nachschlagewerk; es wird sich bei Untersuchungen über die Geschichte einzelner Pflanzen als sehr brauchbares Hülfsmittel erweisen, wie die wenigen, hier angeführten Beispiele darthun.

\section{IV.}

Jules Camus. Studio di lessicografia botanica sopra alcune note manoscritte del Sec. XVI in vernacolo Veneto. (Estr. dal Tomo II, Serie VI degli Atti del R. Istituto Veneto di sc. lett. ed arti.) Venezia 1884. 45 p., $8^{\circ}$.

In einem Exemplare eines 1543 bei Joh. Schott in Strassburg gedruckten, auf Dioscorides ruhenden Kräuterbuches ${ }^{3}$ mit Holzschnitten (von Pritzel in Thesaurus Litteraturae Botanicae, 1872, S. 45, unter No. 1285 aufgeführt), im Besitze des Prof. O. Penzig in Genua, finden sich handschriftliche Randbemerkungen aus jener Zeit in italienischer Sprache, welche die dialectische Färbung der Gegend zwischen Lonigo, Verona und Mantua zeigen. Die Begründung der Annahme dieser Herkunft stützt der Verf. auf zahlreiche,

1) Flückiger 1. c. 634.

2) ebenda 758 .

3) nach Meyer, Geschichte der Botanik, IV. 302, werthloses Machwerk; es fehlt der Strassburger Bibliothek. 
diesen Bemerkungen entnommene Beispiele und auf die zur Erläuterung geeigneten philologischen Schriften der italienischen und deutschen Literatur. Derselbe führt hierauf die erwähnten Bemerkungen zu jeder in dem Druckwerke genannten Pflanze vor und gibt ihre heutigen systematischen Namen an, was sich nur in sehr wenigen Fällen unausführbar erwiesen hat, wo nämlich auch die Abbildungen zu wünschen übrig lassen. Diese Bestimmung der alten italienischen Pflanzennamen ist begreiflicherweise für eingehendere Forschungen über die Geschichte einzelner Heilpflanzen und Nutzpflanzen und die Geschichte der Pharmacie überhaupt nicht ohne Belang.

Wir begnügen uns hervorzuheben, dass der unbekannte Schreiber der Randbemerkungen angibt, Ligustico oder Livisticho wachse in grosser Menge an der genuesischen Riviera und diene in Ligurien statt des Pfeffers gegen Blähungen (ventosità). - Es muss dahin gestellt bleiben, welche Pflanze dieses war, vermutlich nicht unser Levisticum officinale; diese Umbellifere habe ich wenigstens zwischen Spezia und Cannes nirgends getroffen. In wildwachsendem Zustande ist sie überhaupt durchaus unbekannt. ${ }^{1}$

Was ich in Betreff der Wandelungen des altgriechischen Wortes Glycyrrhize in den Sprachen des Mittelalters und der Neuzeit angeführt habe ${ }^{2}$, findet hier, unter dem Artikel Radix dulcis, durch Camus eine weitere Ergänzung.

In einem Aufsatze: Botanique et Philologie, welcher in der "Feuille des Jeunes Naturalistes" in Paris erschienen ist, bespricht Camus eben die Grundsätze, welche ihn bei der Bearbeitung der oben erwähnten Randbemerkungen eines Pflanzenfreundes des XVI. Jahrhunderts geleitet haben, um zu erörtern, in welcher Weise man vernünftiger Weise zu Werke gehen muss, um an der Hand philologischer Kenntnis die Erläuterung der alten Pflanzennamen mit Erfolg unternehmen zu können. Für die französische Sprache hat selbst

1) $\mathrm{Zu}$ diesem Schlusse berechtigen mich die Erkundigungen, welche ich zu diesem Zwecke auch in Ungarn und Siebenbürgen einzog, wobei ich mich, im October 1882, der gütigen Unterstützung Sr. Eminenz des Cardinal-Erzbischofs Dr. Haynald in Kalocsa zu erfreuen hatte. Demnach ist meine bezügliche Angabe in Pharmakognosie, 1883, S. 425 zu berichtigen; niemand kennt Levisticum als wildwachsende Pflanze.

2) Pharmakognosie, S. 353. 
Littré in seinem grossen Wörterbuche gerade diesem Gebiete nicht die genügende Sorgfalt angedeihen lassen.

\section{V.}

LOpera Salernitana "Circa instans" ed il testo primitivo del "Grant Herbier en francoys", secondo due codici del secolo XV, conservati nella regia biblioteca Estense, per Giulio Camus, Professore nella R. Scuolo militare etc. In Modena, coi tipi della Società tipografica. 1886. - 155 Seiten gross $4^{0}$, mit einer Tafel Schriftproben in Heliotypie.

Wer sich mit Pflanzen vertraut machen will, welche im Mittelalter in Südeuropa und Mitteleuropa ihrer Heilwirkung oder sonstigen Nutzens wegen in Ansehen standen, begegnet in Italien, wie in Deutschland besondern Verzeichnissen derartiger Pflanzen. Eines der ehrwürdigsten, welches diesen beiden Ländern zugleich angehört, ist das Capitulare Karl's des Grossen aus dem Jahre 812; ${ }^{1}$ um vielleicht 3 Jahrhunderte später und sehr viel reichhaltiger, weilt geradezu in medicinischer Absicht zusammengestellt, ist das Verzeichnis von Heilpflanzen und Drogen, welches unter dem Namen "Circa instans" aufgeführt wird. Diesem italienischen Verzeichnisse kommt in Betreff der Anzahl der Artikel bei weitem nicht gleich das aus dem Anfange des XV. Jahrhunderts stammende mittelniederdeutsche Arzneibuch aus Gotha ${ }^{2}$ mit 275 Artikeln, welches im übrigen manche gute Anhaltspuncte gewährt und, wie es scheint, an Reichhaltigkeit hinter keinem derartigen Documente des deutschen Mittelalters zurücksteht.

"Circa instans negocium in simplicibus medicinis nostrum versatur propositum ", - unsere Aufgabe bezieht sich jetzt auf die Erörterung der arzneilichen Rohstoffe - , lautet der Eingang des Manuscripts, welcher der hier vorliegenden Ausgabe sogar in heliotypischer Nachbildung der nicht gerade sehr schönen gotischen Schrift beigegeben ist. Und weiter erläutert der Verfasser: „Simplex autem médicina est que talis et qualis est a natura producta". Das Schlagwort "Circa instans" ist in Ermangelung einer Überschrift

1) Flückiger, Pharmakognosie, Berlin 1883. S. 1004.

2) Herausgegeben von Karl Regel, Gotha 1873. 42 Seiten in $4^{\circ}$, Programm des Gymnasium Ernestinum. - Vgl. auch Flückiger, 1. c. 353 647,688 . 
schon seit dem Mittelalter zur Bezeichnung dieser merkwürdigen Liste gebräuchlich; ${ }^{1}$ bisweilen allerdings heisst sie auch Liber de medicina simplici oder Tractatus Herbarum.

Ludwig Choulant, der ausgezeichnete Kenner der Geschichte der Medicin führt aus Circa instans nach den ihm zugänglichen Quellen 273 Nummern, doch ohne weitere Bemerkungen an. ${ }^{2}$ Auch der nicht minder ausgezeichnete Geschichtschreiber der Botanik, Ernst Meyer, ${ }^{3}$ würdigte das Buch Circa instans vollauf nach seiner Bedeutung für die Erlänterung der mittelalterlichen Pflanzenkunde.

Als der Herzog Cesare d'Este im Jahre 1598 von Ferrara nach Modena übersiedelte, gelangte der ausgezeichnete, auch an Handschriften reiche Bücherschatz des in der Kunstgeschichte unvergesslichen Hauses Este nach Modena, wo sich nunmehr die "Biblioteca Estense" als Eigentum der Stadt sorgsamer Pflege zu erfreuen hat. Der Professor an der dortigen Kriegsschule, Giulio Camus, fand 1885 in der Biblioteca Estense zwei mit Malereien gezierte Handschriften des XV. Jahrhunderts auf, deren eine zum ersten Male das Buch Circa instans vollständig gibt, während die andere sich im wesentlichen als eine französische Übersetzung desselben herausstellt, welche mit den Worten beginnt: „En ceste presente besoingne cest nostre propos et entencion de traitier des simples medicines ", also genau wie der lateinische Text. Der französische Übersetzer nennt sich Le petit Pelous und gibt an, seine Arbeit im Jahre 1458 ausgeführt zu haben.

Französische Bearbeitungen des Circa instans waren schon unter dem Namen "Grant herbier" oder "Arbolayre" 4 bekannt, aber Ernst Meyer war es vorbehalten, dieselben als Übersetzungen des Circa instans zu erkennen. ${ }^{5}$ Merkwürdigerweise ist das von Meyer

1) So sagt schon Saladin (Flückiger, Pharmakognosie p. 1017) fol. 3, im Canitel "Libri necessarii ipsi Aromatario": „....... Antidotarium parvum quo omnes comuniter utuntur. Alius etiam liber aliqualiter usualis nominatus Cirea instans et traetat de simplicibus secundum ordinem alphabeti sed parvum est in usu: sed consulo ut quilibet aromatarius penes se habeat et diligenter studeat quoniam in eo inveniet maximam utilitatem."

2) Handbuch der Bücherkunde für die ältere Medicin, Leipzig 1841. 298.

3) Geschichte der Botanik. III (Königsberg, 1856). 511.

4) Flüokiger l. o. p. 987.

5) am angef. Orte. 
benutzte Manuscript aus der Universitätshibliothek zu Königsberg verschwunden, wie Camus sich von dort berichten liess. ${ }^{1}$ Um so verdienstlicher ist es daher, in dem Funde von Modena einen vollständigen Ersatz zu liefern, welcher ohne Zweifel älter ist als der Arbolayre der Pariser Bibliothek; dieser trägt, wie ich mich selbst überzeugte, keine Jahreszahl, wird aber von den Kennern als dem Jahre 1485 angehörig erachtet.

Prof. Camus, der durch eine Reihe von Schriften seine gründliche Bekanntschaft mit der Geschichte der Botanik erwiesen hat, unterzog sich in der vorliegenden. Abhandlung ${ }^{2}$ der Aufgabe, das Buch Circa instans mit der französischen Bearbeitung sorgfältig zu vergleichen, wobei er namentlich auch Gelegenheit zu zahlreichen, interessanten Bemerkungen fand, welche für die Geschichte der französischen Sprache von Belang sein mögen. Diese und eine genauere Beschreibung der Handschriften selbst, der Abkürzungen, welche nicht immer ganz leicht zu entziffern waren, gehen den Auszügen voran, welche Camus den beiden Handschriften entnommen hat.

Die letzteren behandeln ungefähr 500 Pflanzen, der lateinische Text ist mit 470 Miniaturen ausgestattet, von denen allerdings kaum 50 bescheidenen künstlerischen Anforderungen einigermassen gerecht werden. Die Bilder des französischen Theiles oder "Grant Herbier" sind, wenn auch einförmiger, doch im ganzen besser ausgeführt, entsprechen aber keineswegs immer dem Texte, sondern stellen mitunter ganz andere Dinge vor. Der Herausgeber weist ferner nach, dass "Le petit Pelous", der Übersetzer, in welchem Camus einen französischen, in Italien studirenden Mediciner zu vermuten geneigt ist, sich keineswegs genau an das Buch Circa instans gehalten, sondern bisweilen etwas weggelassen, oft auch anderes beigefügt hat. Die Schriftzeichen sind in der französischen Bearbeitung gleichfalls ziemlich unschöne, gotische Buchstaben, welche jedoch offenbar nicht von der gleichen Hand herrühren, wie diejenigen des lateinischen Circa instans oder Tractatus Herbarum. Hingegen hat Le petit Pelous noch ein grosses, über 4000 Artikel umfassendes Synonymen-Verzeichnis beigefügt, welches wahrscheinlich auf älteren Zusammen-

1) Seite 11 des vorliegenden Buches.

2) Abdruck aus Vol. IV, Serie II der Memorie della R. Accademia di Seienze, Lettere ed Arti di Modena, Sezione di Lettere, p. 49 e seg. 
stellungen beruht. Dasselbe bezieht sich grösstentheils auf Arzneipflanzen, bringt aber auch hier und da der Medicin oder Pharmacie ganz fremde Wörter und bietet dergleichen nicht nur aus der lateinischen, sondern auch aus der griechischen und arabischen Sprache. Obwohl erläuternde Bemerkungen in diesem grossen SynonymenVerzeichnisse fehlen oder doch nur sehr kurz gehalten sind, leistet es doch wie begreiflich, gute Dienste bei der Deutung alter Pflanzennamen und würde wohl einer vollständigen Bearbeitung wert sein. Dieses zeigt schon die von Camus hier mitgeteilte Auswahl, woraus sich aber auch erkennen lässt, dass der Herausgeber dieser Synonymen jedenfalls mit umsichtiger Kritik an sein Werk gehen musste.

Aus dem Tractatus Herbarum oder Circa instans hat $\mathrm{Camus}$ 508 Artikel ausgezogen und denselben jedesmal die französische Übersetzung beigefügt, wie Le petit Pelous sie verfasst hat. Überall sind ferner die Namen der Pflanzen nach der gegenwärtigen Systematik in Anmerkung angeführt oder doch wenigstens in möglichster Wahrscheinlichkeit gedeutet, wo Gewissheit nicht zu erlangen war. In Betreff der Namen, welche Camus beibringt, wird man hier und da wohl anderer Ansicht sein können, sicherlich aber hat derselbe in der weitaus grössten Zahl der Fälle die Wahrheit zu ermitteln verstanden, so dass seine Arbeit für die Geschichte der Pharmakognosie von erheblichem Werte bleibt; die Benutzung derselben wird durch zwei gute Register sehr erleichtert.

No. 32, Seite 36 dürfte Antalis wohl jenes schon bei Myrepsus ${ }^{1}$ vorkommende Dentalium oder Entalium bedeuten, worüber z. B. noch Schröder ausführlich berichtete. No. 72, Seite 43,

1) Flückiger, Pharmakognosio 1009.

2) Pharmacopoeia medico-chymica. Olm 1649. V. 328 :

„Dentalium est conchula parva, oblonga, exterius asperula, intus levissima, tubuli modo concava, alteraque parte rimam habens canini dentis instar acuminatam, nude ef dentalium nominatur.

N. Non piscis, sed vermis oblongus, ac tenuis dentalii incola est; adnascitur saxis marinis, conchisve vetustis.

Entalium ibidem testaceum quoddam est marinum, instar parvi cornu longiusculum, rectum et cavum, foris striatum, intus laeve, raro longitudinem digiti superans."

Heute bilden die Dentalien die höchst eigentümliche Gruppe der Scaphopoda, welche auch in den Tiefen der europäischen Meere zu treffen sind. - Vergl. z. B. Claus, Grundzüge der Zoologie. 1882. 27. 
F. A. Flückiger, Italien. Beiträge z. Gesch. d. Pharmacie u. Botanik. 689

Blatte bisantie; diese merkwürdige, ebenfalls dem Thierreiche entnommene Droge habe ich in meinen Documenten zur Geschichte der Pharmacie ${ }^{1}$ erläutert.

Bei No. 336, Seite 97, verwirft Camus die Vermutung, dass Nux vomica der Circa instans der Same von Strychnos Nux vomica gewesen sein könnte. Dagegen lässt sich immerhin noch eine Stelle aus Serapion anführen, die nicht ohne Wahrscheinlichkeit doch auf die heutige Nux vomica gedeutet werden mag. ${ }^{2}$ Dass diese zur Zeit Serapion's, im XI. oder XIII. Jahrhundert, der arabischen Medicin wirklich bekannt war, lässt sich annehmen, weil z. B. die deutschen Apotheken mindestens im Jahre 1500 die Strychnos - Samen führten. ${ }^{3}$

Bei No. 420, Seite 114, wird Sarcocolla wie gewöhnlich der nur dem Namen nach bekannten Penaea mucronata L. zugeschrieben. Die chemische Natur und die Herkunft der Sarcocolla sind noch nicht aufgeklärt, aber alles spricht dafür, darin eine Art Traganth zu erblicken und die Droge von einer Papilionacee abzuleiten." Penaea kann schon deswegen nicht in Betracht kommen, weil z. B. nach Bentham et Hooker, Genera Plantarum III (I, 1880) 202, die sämtlichen 9 Arten des Genus Penaea in Südafrika einheimisch sind, ebenso die 9 oder 10 Species Sarcocolla.

Zu No. 448, Seite 119, sei die Bemerkung gestattet, dass Hanbury und ich keineswegs Solatrum rusticum für Atropa Bellad o n na erklärten, sondern vielmehr geneigt sind, diese mit Camus (Nr. 468, Seite 123) in Strignum zu erkennen, wie aus unsern weitern Beitrügen zur Geschichte der Belladonna ${ }^{5}$ zur Genüge hervorgeht. Im Arbolayre der Pariser Bibliothek steht bei Solatrum rusticum ausdrücklich das Synonym Alkakengi.

1) Archiv der Pharmacie 205 (1876); Seite 55 des Separatabdruckes, Halle, 1876.

2) Flückiger, Pharmakognosie 963.

3) Flückiger, Pharmakognosie 963 (nach einer Mitteilung von Prof. Schär).

4) Dymock, Materia medica of Western India. Bombay 1886. 290.

5) Pharmacographia, London 1879 p. 456 . - Flückiger, Die Frankfurter Liste, Archiv der Pharmacie 201 (1872) 509 und Pharmakognosie p. 668. 